\title{
GLACIER SURFACE MONITORING BY MAXIMIZING MUTUAL INFORMATION
}

\author{
Esra Erten $^{a}$, Cristian Rossi $^{b}$, Irena Hajnsek $^{c, d}$ \\ ${ }^{a}$ ITU, Civil Engineering Faculty, Department of Geomatic Engineering \\ 80626 Maslak Istanbul, Turkey \\ ${ }^{b}$ DLR, German Aerospace Center, Remote Sensing Technology Institute, \\ D-82234 Wessling, Germany \\ ${ }^{c}$ ETH Zurich, Institute of Environmental Engineering, Earth Observation and Remote Sensing Group \\ CH-8093 Zurich, Switzerland \\ ${ }^{d}$ DLR, German Aerospace Center, Microwaves and Radar Institute \\ D-82234 Wessling, Germany
}

Commission VII/2

KEY WORDS: SAR, polarimetry, information theory, glacier

\begin{abstract}
:
The contribution of Polarimetric Synthetic Aperture Radar (PolSAR) images compared with the single-channel SAR in terms of temporal scene characterization has been found and described to add valuable information in the literature. However, despite a number of recent studies focusing on single polarized glacier monitoring, the potential of polarimetry to estimate the surface velocity of glaciers has not been explored due to the complex mechanism of polarization through glacier/snow. In this paper, a new approach to the problem of monitoring glacier surface velocity is proposed by means of temporal PolSAR images, using a basic concept from information theory: Mutual Information (MI). The proposed polarimetric tracking method applies the MI to measure the statistical dependence between temporal polarimetric images, which is assumed to be maximal if the images are geometrically aligned. Since the proposed polarimetric tracking method is very powerful and general, it can be implemented into any kind of multivariate remote sensing data such as multi-spectral optical and single-channel SAR images.

The proposed polarimetric tracking is then used to retrieve surface velocity of Aletsch glacier located in Switzerland and of Inyltshik glacier in Kyrgyzstan with two different SAR sensors; Envisat C-band (single polarized) and DLR airborne L-band (fully polarimetric) systems, respectively. The effect of number of channel (polarimetry) into tracking investigations demonstrated that the presence of snow, as expected, effects the location of the phase center in different polarization, such as glacier tracking with temporal HH compared to temporal VV channels. Shortly, a change in polarimetric signature of the scatterer can change the phase center, causing a question of how much of what I am observing is motion then penetration. In this paper, it is shown that considering the multi-channel SAR statistics, it is possible to optimize the separate these contributions.
\end{abstract}

\section{INTRODUCTION}

Polarimetric Synthetic Aperture Radar (PolSAR) data are being used more and more for temporal analysis such as crop inventory, change detection, land use management and etc. Although there are interesting works in glacier monitoring with single-channel single-polarized SAR images (Erten et al., 2009, Reigber et al., 2008, Fallourd et al., 2011), the potential of PolSAR in glacier monitoring has not been fully investigated yet. Indeed, a change in the polarimetric signature affects the phase center in the presence of the snow, meaning that tracking with temporal HH channels may differ from the VV one (Rott and Davis, 1993). To take into account this polarimetric information into the tracking, instead of single channel tracking, as in common in literature, a new polarimetric approach is proposed in this paper. The approach proposed in this work for tackling this problem is the maximization of the Mutual Information (MI) between temporal polarimetric covariance matrices, yielding the best fit between different displacement (shift) vectors.

The presentation of the paper is as follows. Section 2 addresses the theoretical background of the multi-channel MI and express the proposed algorithm how to use in glacier monitoring. Section 3 is dedicated to experimental results on for temporal glacier monitoring with DLR airborne E-SAR sensor and Envisat images, presenting the performance of the proposed algorithm in a long and short wavelength, respectively. Section 4 concludes the work with discussions.

\section{GLACIER SURFACE MONITORING}

This paper expands the ideas firstly presented in (Erten et al., 2012), which uses the MI to characterize the temporal scene in terms of PolSAR images. In this work, the mutual information, which is maximum if the temporal images are geometrically aligned, is used for multi-channel (PolSAR) tracking by exploiting the second order statistics of the acquisitions.

\subsection{Theoretical Background}

A temporal acquisition vector, for each pixel, $\vec{k}=\left[\vec{k}_{1} \vec{k}_{2}\right]^{T}$ is a complex vector distributed as a multi-component circular Gaussian $\mathcal{N}^{C}(0, \Sigma)$ that consists of two target vectors $\vec{k}_{1} \sim$ $\mathcal{N}^{C}\left(0, \Sigma_{11}\right)$ and $\vec{k}_{2} \sim \mathcal{N}^{C}\left(0, \Sigma_{22}\right)$ obtained from temporal multi channel SAR images at time $t_{1}$ and $t_{2}$, respectively. Here, the number of elements in one of the target vectors $\vec{k}_{i}$ at time $t_{i}$ is represented by $m$, and hence the temporal target vector $\vec{k}$ has the dimension of $q=2 \times m$. For example, $q=2$ corresponds to interferometric SAR (InSAR) images whereas $q=6$ corresponds polarimetric interferometric (PolInSAR) images. It can be remarked that with single channel data $(m=1)$, only one copolarized channel $\vec{k}_{i}=\vec{k}_{h h}$ or $\vec{k}_{i}=\vec{k}_{v v}$ is recorded, and the phase carries no useful information for distributed targets. When multichannel (polarimetric) data are available e.g. $\vec{k}_{i}=\left[k_{h h} k_{h v} k_{v v}\right]$, phase differences between channels provide information about 
dielectric and geometric properties of the scattering medium (Lee and Pottier, 2009).

The true covariance matrix $\Sigma$, which contains sufficient statistics to characterize the acquisition vector $\vec{k}$, is not known, and is estimated using a maximum likelihood method by $n$-sample $(n$ look) spatial coherent averaging: $A=\frac{1}{n} \sum_{j=1}^{n} \vec{k}_{j} \vec{k}_{j}^{\dagger}$. The true covariance matrix $\Sigma$, as well as its $n$-sample estimate $A$, can be portioned as:

$$
\Sigma=\left[\begin{array}{ll}
\Sigma_{11} & \Sigma_{12} \\
\Sigma_{21} & \Sigma_{22}
\end{array}\right] \text { and } A=\left[\begin{array}{cc}
A_{11} & A_{12} \\
A_{21} & A_{22}
\end{array}\right]
$$

which summarize all the information (the joint and marginal) from temporal multi-channel SAR acquisitions. $A_{12}=A_{21}^{\dagger}$ is a $m \times m$ dimensional cross correlation matrix between the acquisition vectors $\vec{k}_{1}$ and $\vec{k}_{2}$ which characterizes the interferometric and polarimetric information. Since the variables fall naturally into two sets, they can be correlated or uncorrelated processes $\left(0 \leq P^{2}=\Sigma_{11}^{-1} \Sigma_{12} \Sigma_{22}^{-1} \Sigma_{21} \leq I_{m}\right)$ over time depending on the monitored objects. The matrices $A_{11}$ and $A_{22}$ are the standard $n$-look and $m \times m$ dimensional polarimetric covariance matrices of separate temporal images. Note that, for $m=1$, matrix $A_{i i}$ reduces to the single intensity scalar $a_{i}$.

\subsection{Proposed Algorithm:}

The MI is well known technique for co-registration of remotely sensed images such as medical, optical and radar images (Maes et al., 1997, Reinartz et al., 2011, Chen et al., 2003). In this paper, the MI is adopted for glacier monitoring in terms of temporal multi-channel images. The major requirement to compute the MI between two images is the accurate estimation of the joint histogram from the samples. However, in the case of polarimetric images, it is really time consuming work due to the requirement of 6 dimensional joint histogram. To overcome this problem joint distribution between temporal polarimetric covariance matrices derived by Erten et al., 2012 is used.

In probability and information theory, the mutual information is a commutative measure of the difference between the joint probability distribution $\mathrm{p}_{X, Y}(x, y)$ and the marginal probability distributions $\mathrm{p}_{X}(x)$ and $\mathrm{p}_{Y}(y)$ of the random variables $X$ and $Y$, respectively (Papoulis, 2002). Taking the joint distribution $\mathrm{p}\left(A_{11}, A_{22}\right)$ of temporal polarimetric covariance matrices $A_{11}$ and $A_{22}$, the MI between them due to the Wishart process in time is (Erten et al., 2012):

$$
\begin{aligned}
\mathcal{D}_{M I}\left(A_{11} ; A_{22}\right) & =E\left\{\log \left({ }_{0} \tilde{F}_{1}(n, C)\right\}\right. \\
& -n \log \left(I_{m}-P^{2}\right)-\frac{2 n P^{2}}{I_{m}-P^{2}} \\
C & =\Sigma_{11 \mid 22}^{-1} \Sigma_{12} \Sigma_{22}^{-1} A_{11} A_{22} \Sigma_{22}^{-1} \Sigma_{21} \Sigma_{11 \mid 22}^{-1} \\
\Sigma_{11 \mid 22} & =\Sigma_{11}-\Sigma_{12} \Sigma_{22}^{-1} \Sigma_{21}
\end{aligned}
$$

where ${ }_{0} \tilde{F}_{1}(n, C)$ is the complex hypergeometric function of matrix $C$. This function can be calculated with the help of the positive eigenvalues of the $m \times m$ Hermitian matrix $C$ by (Smith and Garth, 2007).

In terms of tracking applications the best match between temporal images is found by maximizing a tracking algorithm between a reference image at time $t_{1}$ and a second image at time $t_{2}$. In practice, the reference image is fixed, and the second image is shifted by a factor of resolution (subpixel). At every subpixel shift the value of the tracking algorithm is stored, and the subpixel shift at the peak is assumed to be the correct displacement between images. In this work, the maximization of the MI is used as a tracking algorithm.

Let's assume that a reference block $A_{11_{s}}=\left[A_{11_{1}}, A_{11_{2}}, \cdots\right.$ $\left.A_{11_{s-1}}, A_{11_{s}}\right]^{T}$ matches a second block $A_{22_{i s}}=\left[A_{22_{i 1}}, A_{22_{i 2}}\right.$, $\left.\cdots, A_{22_{i(s-1)}}, A_{22_{i s}}\right]^{T}$. This matching results in a displacement (shift) vector $\vec{v}_{i}$, where $i$ indicates the block which is shifted $i$ subpixels, and $s$ is the number of pixels in block $A_{11_{s}}$ and $A_{22_{i s}}$. Then, the displacement vector $\vec{v}$ between the images is obtained by maximizing the $\operatorname{MI}\left(A_{11_{s}}, A_{22_{i s}}\right)$ for each block $i$

$$
\vec{v}=\underbrace{\operatorname{argmax}}_{\vec{v}_{i}} f\left(\operatorname{MI}\left(A_{11_{s}}, A_{22_{i s}}\right), \vec{v}_{i}\right) .
$$

\section{EXPERIMENTAL RESULTS}

This section contains a concise presentation of the proposed polarimetric tracking method. It is not intended to give an in-depth geophysical analysis, but merely an example of motion estimation using the MI. To validate the proposed approach and to show its performances in different acquisition circumstances, two different sensors having different system parameters have been used. It is worth mentioning that the precise/coherent geometric coregistration of temporal SAR images is a strict requirement for tracking algorithms. In order to perform a precise coregistration for spaceborne image, backward geocoding was employed (Sansosti et al., 2006). In the backward geocoding approach, for each DEM element, the image pixel with the nearest range-Doppler coordinate is calculated. For airborne images, which is more challenge than the spaceborne ones due to the absence of precise orbit information, the multi-squint approach has been applied (Prats et al., 2009).

The first pair considers airborne images acquired by the Experimental SAR (E-SAR) system of the German Aerospace Center (DLR) in the frame of the SWISAR campaign, 2006 (Prats et al., 2009). Table 1 summarizes the acquisitions over the Aletsch glacier, located in Swiss Alps including their system parameters. This pair, having one day temporal resolution, provides an example of monitoring the fast glacier surface velocity in the presence of relatively correlated speckle patterns.

To address the application potential of the proposed method in single channel SAR images, spaceborne sensor ASAR Envisat images acquired over Inyltshik glacier located in Kyrgyzstan are processed. In case of spaceborne monitoring, a very fast flowing glaciers deserves a special attention. In particular, the speckle patterns of the temporal images are no longer correlated due to the satellite fixed temporal resolution (35 days for Envisat) and the shorter wavelength.

Fig. 1 shows the histograms of the coherences of both pairs in the glacier area. The decorrelation effect in the Envisat pair compared to the E-SAR pair is already visible in the figure. In case of fast moving glacier, a larger wavelength like the L-band (24.3 $\mathrm{cm})$ one, compared to a shorter wavelength X-band $(5.6 \mathrm{~cm})$, tackles some of the decorrelation problems because of its properties to penetrate more into the snow and the firn.

\subsection{Aletsch glacier (coherent) monitoring with L-band}

This section presents the results to show the performances of the proposed fully polarimetric MI approach with L-band data having one day temporal resolution. In particular, a detailed analysis of polarimetric tracking based on MI over glacier having temporal (relatively) correlated speckle patterns is handled. Fig. 2 plots the 
Table 1: SAR acquisitions

\begin{tabular}{|l|c|c|}
\hline & Envisat C-band & E-SAR L-band \\
\hline temporal resolution & 35 day & 1 day \\
\hline wavelength & $3.1 \mathrm{~cm}$ & $24.3 \mathrm{~cm}$ \\
\hline along-track resolution & $7.8 \mathrm{~m}$ & $1.5 \mathrm{~m}$ \\
\hline across-track resolution & $4 \mathrm{~m}$ & $3 \mathrm{~m}$ \\
\hline polarization & $\mathrm{VV}$ & $\mathrm{HH}, \mathrm{VV}, \mathrm{HV}, \mathrm{VH}$ \\
\hline baseline & $360 \mathrm{~m}$ & $0 \mathrm{~m}$ \\
\hline
\end{tabular}

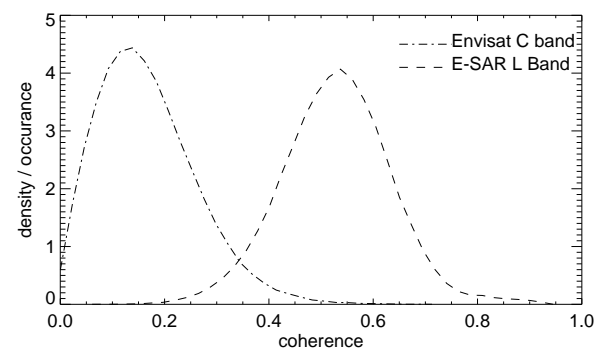

Figure 1: Reconstructed coherence histograms corresponding to the Aletsch glacier, after a 1 day and to Inyltshik glacier after 35 day time interval, using a coherence estimation window of $11 \times 11$ samples.

estimated along-track (a) and the across-track (b) displacements through two SWISAR campaign acquisitions. In here, the amplitude of the displacements is the division of the displacements vector by the time interval between acquisitions. The alongtrack information turns out to be very valuable, since in some areas the across-track (LOS) measurement cannot yield any displacement due to the lack of movement in the across-track direction, highlighting the limitation of DInSAR application. The amplitude of the displacements in both directions can be easily discriminated because of the geometrical characteristics of the monitored glaciers. Considering previous in situ measurements, the displacement maps are found correctly in both across-track and along-track directions. The first one with the average of 17 $\mathrm{cm} /$ day and the other one with the average speed of $36 \mathrm{~cm} /$ day, respectively.

To test the performances of the MI based tracking through whole glacier, the classical maximum likelihood estimation performance criteria:

$$
Q=\frac{\max (M I)-\operatorname{mean}(M I)}{\operatorname{mean}(M I)-\min (M I)}
$$

has been plotted in Fig. 2(c). It can be easily seen that areas including moving ice blocks, crevasses and rocks have a significantly better estimation performance than areas including layers of snow. Note that a detailed analysis of Aletsch glacier surface velocity monitoring with single channel SAR (only temporal $V V$ channels) can be found in (Prats et al., 2009) and (Reigber et al., 2008).

\subsection{Inyltshik glacier (incoherent) monitoring with C-band}

As final example for the MI based tracking, the surface displacement map of Inyltshik glacier by single channel Envisat C-band, highlighting the application potential of the proposed polarimetric tracking in decorrelated data set (see Fig. 3(a)), has been obtained. The surface velocity map -Fig. 3(b)-, which is in accordance with previous in situ measurements, shows the typical surface velocity pattern of glaciers with an highest velocities in the center of the glacier and a strong gradient towards the boundary. A more detailed study about the geophysical properties of the

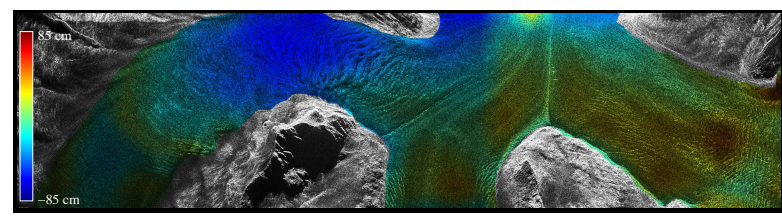

(a) Along-track displacement

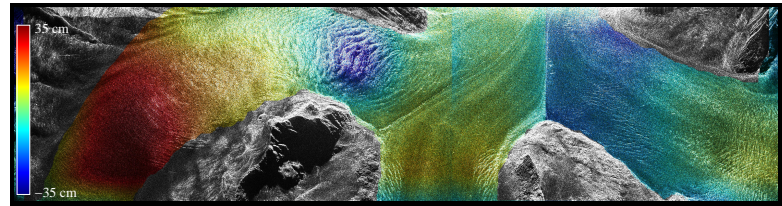

(b) Across-track displacement

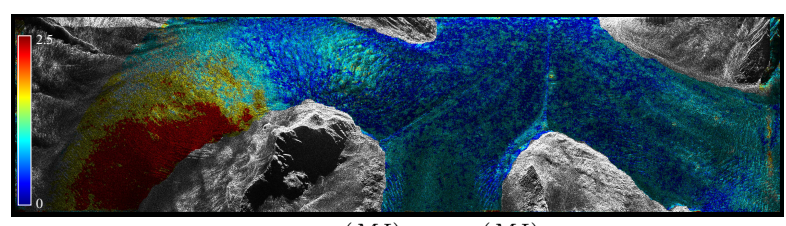

(c) $\frac{\max (M I)-\operatorname{mean}(M I)}{\operatorname{mean}(M I)-\min (M I)}$

Figure 2: Retrieval of the along-track (a) and across-track (b) displacements, after a 1 day time interval, using the proposed polarimetric tracking. (c) The robustness map. The horizontal direction corresponds to the along-track whereas the vertical direction corresponds to the across-track direction.

surface velocity of the Inyltshik glacier can be found in (Mayer et al., 2008, Erten et al., 2009).

\section{CONCLUSIONS AND DISCUSSIONS}

Multi-channel (polarimetric) tracking approach has been demonstrated to estimate $2-\mathrm{D}$ glacier surface velocity by maximizing the MI between temporal acquisitions. Two conclusions can be made after analyzing the first results in multi-channel tracking. Firstly, in the ablation zone of the glacier, the multi-channel tracking improves the surface velocity estimation significantly. This can be explained thanks to the natural properties of the ice blocks and coherent scatters in this area. Since polarimetry does not have a significant role in those features, making use of the multi-channel -hence polarimetric channels, increase the randomness of the acquisitions, and so the quality of the estimation. Secondly, in the accumulation zone of the glacier, tracking between interferometric methods with different polarization can cause a misleading information based on the difference in penetration depth of $\mathrm{HH}$ and $V V$ channels into snow. It has also been shown that thanks to multi-channel tracking this misleading information can be optimized since the movement/shift between each temporal polarimetric channel is the same. However, availability of ground measurement is required for validating the proposed method. The following specific research questions will be answered with ground measurements:

- At what level of accuracy can glacier surface velocity can be measured thanks to POLSAR images?

- Can PolSAR glacier surface velocity measure improve the one with single-channel single-polarized SAR images.

- How can the proposed algorithm be depended on the wavelength (penetration to ice)? 


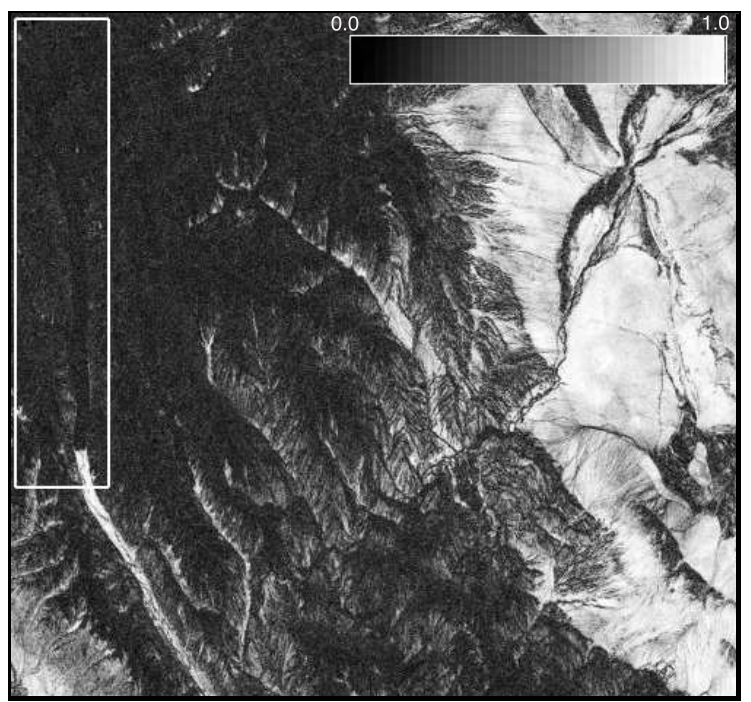

(a) Coherence image

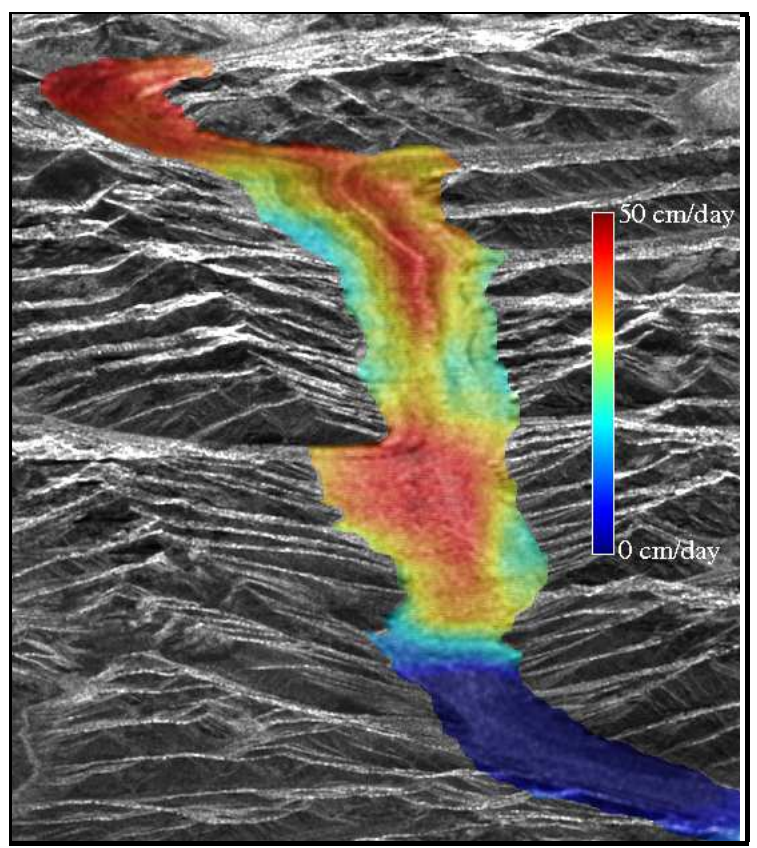

(b) Displacement map

Figure 3: (a) Interferometric coherence image of Envisat scene. The white rectangle indicates the location of Inyltshik glacier. (b) Velocity map $\sqrt{d_{a l}^{2}+d_{a c}^{2}}$ produced by maximizing the MI, where $d_{a l}$ and $d_{a c}$ shows the displacement vector in along-track and across-track direction, respectively.

\section{ACKNOWLEDGEMENTS}

The authors would like to thank Pau Prats and the German Aerospace Center (DLR) for providing interferometric process of E-SAR Lband images, and Deutsches GeoForschungsZentrum (GFZ) in Potsdam for providing the ENVISAT data sets of Inyltshik glacier region.

\section{REFERENCES}

Chen, H. M., Varshney, P. K. and Arora, M. K., 2003. Performance of mutual information similarity measure for registration of multitemporal remote sensing images. Geoscience and Remote Sensing, IEEE Trans. on 41(11), pp. 2445-2454.
Erten, E., Reigber, A., Ferro-Famil, L. and Hellwich, O., 2012. A new coherent similarity measure for temporal multi-channel scene characterization. Geoscience and Remote Sensing, IEEE Trans. on 50(7), pp. 1-13.

Erten, E., Reigber, A., Hellwich, O. and Prats, P., 2009. Glacier velocity monitoring by maximum likelihood texture tracking. Geoscience and Remote Sensing, IEEE Trans. on 47(2), pp. 394 405 .

Fallourd, R., Harant, O., Trouvé, E., Nicolas, J. M., Gay, M., Walpersdorf, A., Mugnier, J. L., Serafini, J., Rosu, D., Bombrun, L., G., V., Cotte, N., Vernier, F., Tupin, F., Moreau, L. and Bolon, P., 2011. Monitoring temperate glacier displacement by multitemporal terrasar-x images and continuous gps measurements. Selected Topics in Applied Earth Observations and Remote Sensing, IEEE Journal of 4(2), pp. 372-386.

Lee, J. and Pottier, E., 2009. Polarimetric radar imaging: from basics to applications. CRC.

Maes, F., Collignon, A., Vandermeulen, D., Marchal, G. and Suetens, P., 1997. Multimodality image registration by maximization of mutual information. Medical Imaging, IEEE Trans. on 16(2), pp. 187-198.

Mayer, C., Hagg, W., Lambrecht, A., Helm, A. and K., S., 2008. Postdrainage ice dam response at lake merzbacher, inylchek glacier, kyrgyzstan. Geografiska Annaler 90(1), pp. 8796.

Papoulis, A., 2002. Probability, random variables, and stochastic processes. Fourth edn, McGraw-Hill, Inc., Singapore.

Prats, P., Scheiber, R., Reigber, A., Andres, C. and Horn, R., 2009. Estimation of the surface velocity field of the aletsch glacier using multibaseline airborne sar interferometry. Geoscience and Remote Sensing, IEEE Trans. on 47(2), pp. 419-430.

Reigber, A., Prats, P., Scheiber, R., Andres, C., Erten, E. and Hellwich, O., 2008. Vermessung der fliegeschwindigkeit alpiner gletscher mit flugzeuggesttzter differentieller sar interferometrie. Allgemeine Vermessungs-Nachrichten (AVN) pp. 419-430.

Reinartz, P., Muller, R., Schwind, P., Suri, S. and Bamler, R., 2011. Orthorectification of vhr optical satellite data exploiting the geometric accuracy of terrasar-x data. ISPRS Journal of Photogrammetry and Remote Sensing 66, pp. 124-132.

Rott, H. and Davis, R. E., 1993. Multifrequency and polarimetric sar observations on alpine glaciers. Annals of glaciology 17, pp. 98-98.

Sansosti, E., Berardino, P., Manunta, M., Serafino, F. and Fornaro, G., 2006. Geometrical sar image registration. Geoscience and Remote Sensing, IEEE Trans. on 44(10), pp. 28612870.

Smith, P. J. and Garth, L. M., 2007. Distribution and characteristic functions for correlated complex Wishart matrices. Journal of Multivariate Analysis 98(4), pp. 661-677. 